Accepted (peer-reviewed) version of article

It is authorized for self-archiving after an embargo period of 24 months.

Formatted by the author to enhance readability. 
Published in The evolution of primate social cognition, edited by L. D. di Paolo, F. di Vincenzo, \& F. de Petrillo (pp. 299-326), Springer (2018).

\title{
Visuospatial integration: Paleoanthropological and archaeological perspectives
}

\author{
Emiliano Bruner ${ }^{1}$, Enza Spinapolice ${ }^{2}$, \\ Ariane Burke ${ }^{3}$, Karenleigh A. Overmann ${ }^{4}$ \\ ${ }^{1}$ Corresponding author: Centro Nacional de Investigación sobre la Evolución Humana, Paseo Sierra de \\ Atapuerca 3, 09002 Burgos, Spain. Email: emiliano.bruner@cenieh.es \\ ${ }^{2}$ La Sapienza Università di Roma, Roma (Italia) \\ ${ }^{3}$ Université de Montreal (Canada) \\ ${ }^{4}$ Center for Cognitive Archaeology, University of Colorado, Colorado Springs (USA)
}

\begin{abstract}
The visuospatial system integrates inner and outer functional processes, organizing spatial, temporal, and social interactions between brain, body, and environment. These processes involve sensorimotor networks like the eye-hand circuit, which is especially important to primates, given their reliance on vision and touch as primary sensory modalities and the use of the hands in social and environmental interactions. At the same time, visuospatial cognition is intimately connected with egocentric memory, self-awareness, and simulation capacity. In the present article, we review issues associated with investigating visuospatial integration in extinct human groups through the use of anatomical and behavioral data gleaned from the paleontological and archaeological records. In modern humans, paleoneurological analyses have demonstrated noticeable and unique morphological changes in parietal cortex, an area crucial to visuospatial management. Archaeological data provides information on hand-tool interaction, the spatial behavior of past populations and their interaction with the environment (e.g. in domains like landscape use and navigation, the spatial relations implicit in social networks, etc.). Visuospatial integration may represent a critical bridge between extended cognition, self-awareness, and social perception. As such, visuospatial functions are relevant to the hypothesis that human evolution is characterized by changes in brain-body-environment interactions and relations, which enhance possibilities for integrating inner and outer cognitive components through neural plasticity and a specialized embodiment capacity. We therefore advocate the investigation of visuospatial functions in past populations through the paleoneurological study of anatomical elements and archaeological analysis of visuospatial behaviors.
\end{abstract}

\section{Visuospatial evolution}

Visuospatial functions have been long considered to be strictly mechanical phenomena. Activities like "handling” and "walking," for example, have been viewed as automatic processes involving sensory feedbacks and muscle adjustments (i.e., as processes that do not involve high-cognitive loads and which have only limited associations with more complex cognitive functions like memory, executive functions, and consciousness). This view of visuospatial functions has persisted, despite the famous "cybernetic paradox" that we can build computers capable of performing calculations that far exceed human computational powers, but we have not still been able to design a machine able to walk with fluidity. Interest in visuospatial processes has been renewed in the last decade because of an increasing attention towards parietal lobes, which include crucial areas for visuospatial management. Converging evidence from evolutionary neuroanatomy, functional neuroimaging, brain 
modeling, and cognitive sciences, suggests that visuospatial integration may be profoundly intermingled with our most complex cognitive processes (Bruner and Iriki 2016).

The term "visuospatial integration" can be rather vague, since it embraces a variety of concepts and encompasses multiple functional aspects, and it can mean different things in neurobiological, psychological, and philosophical contexts. These subtle but relevant differences in definitions and concepts can generate misunderstandings or cultural barriers between disciplines, and this can be particularly detrimental for a topic which is clearly multidisciplinary. The word "visuospatial" conjoins concepts associated with visual perception, including the physiological mechanisms of vision and the associated neural codes and networks, with spatial perception, a basic perceptual relation between an organism and its environment. Both vision and spatiality have a physical component, as well as a "virtual" one involved in mental imagery. For its part, the word "integration" denotes functionality in combining multisensory inputs into a holistic perceptual experience.

Beyond any definition, visuospatial integration is generally something difficult to test in modern populations because of its involvement in very distinct tasks, ranging from basic spatial management to social perception. Visuospatial cognition in extinct human species is even more difficult to evaluate, because it must be detected and quantified indirectly through the partial and fragmentary information provided by the paleontological and archaeological records. Paleontology is concerned with the bones of past populations, archaeology with their material relics. Accordingly, visuospatial functions in human evolution must be inferred from the visuospatial elements and behaviors revealed by bone anatomy and cultural cues. Interpreting this indirect evidence can be challenging, as most biological and cultural processes leave no traces and thus remain largely invisible to the paleontological and archaeological records. Nonetheless, a proper evaluation of these biological and cultural remnants represents the only chance to test human cognitive evolution directly on the species involved in the phylogenetic process. As for other cognitive capacities, some visuospatial behavioral changes will remain silent to the paleontological and archaeological analysis, but others may have left some physical indications. Certainly, any cognitive evaluation on extinct species or populations must be framed into a broader context, in which neurobiological and behavioral data on extant groups support a general comparative and functional perspective.

Cognitive archaeology has directly addressed the evolution of spatial cognition since the late 1980s, through the pioneering work of Thomas Wynn (1989). Wynn suggested that bilateral symmetry in early Acheulean bifaces implied that the hominids who produced them about 1.8 million years ago (Mya) had developed an ability to coordinate shape and spatial information, "arguably the initial step in the evolutionary sequence of modern visuo-spatial integration” (Wynn 2014:291; also see Wynn 2002). Similarly, by roughly 500 thousand years ago (Kya), the use of complex prepared core techniques and the imposition of 3D symmetry in late Acheulean bifaces suggested that there were further evolutionary developments in visuospatial integration, including the capacities for "inner virtual space” (Bruner and Lozano 2014:274) and landscape mental mapping (Wynn 2000). By 50 Kya, behavioral and morphometric change in Homo sapiens included greater use of projectile weapons (Lombard and Haidle 2012) and possibly a more specialized eye-hand visuospatial interface (Bruner and Lozano 2014). More recently, following the idea that cognition is embodied, embedded, extended, and enacted (see below), cognitive archaeology has begun 
evaluating whether such behavioral and morphological changes might signal the emergence of additional developments in visuospatial capacity, enabling in our species "a more pervasive prosthetic adaptation” (Overmann 2015:165).

\section{Beyond neurons}

Ideas about how the physical body and material environment might influence, aid, and perhaps even constitute human cognition have been introduced in recent decades. Cognition has not remained localized in brain form and function (the neurocentric view of cognition) or viewed as a computational process involving perceptual inputs, computer-like manipulation, and motor outputs (the computational model of cognition). Rather, it has become embodied (the body has a role in cognition-Lakoff and Johnson 2008; Prinz 2009), situated (cognition is influenced or perhaps even constituted by things external to the brainRobbins and Aydede 2009), embedded in (cognition is influenced by aspects of the environment-Smith 1999) and distributed across (cognition involves resources and processes in networks of interacting agents and objects-Hutchins 1995) environments containing natural, social, and culturally modified material resources. Beyond distribution, cognition may be extended, the notion that physical, social, and material resources may be at least partially constitutive of cognition, perhaps even on par with psychological processing and neural activity, though the ways in which brain-external resources contribute undoubtedly differ from brain-internal ones (Clark and Chalmers 1998; Clark 2008).

The idea that physical and material elements may be included within the boundaries of cognition is especially relevant to human cognitive evolution, given the unparalleled degree and extent to which our species uses material culture. Historical ideas about brain functionality have also been updated with insights gained from research in Artificial Intelligence (AI). For example, traditional cognitive models have proposed that brains recreate the external world in the form of internal representations. However, breakthroughs in AI research have suggested that highly representational cognition would be inefficient and likely evolutionarily unsustainable; internal representations would also be redundant to the information-bearing capacity of the world itself, information that an organism obtains through interactivity with the world (Clark 1997).

The turn to anti-representationalism helped inspire the idea that cognition is enactive, constituted by the dynamic interactivity between brains, bodies, and material environments (Hutto and Myin 2013; Malafouris 2013). Thinking and doing are not separable. Performative skill, such as that involved in complex flintknapping techniques (e.g. Levallois), can therefore be viewed as mastering the enactivity of variable domains (i.e. psychological processes, motor movements, and material properties) to produce invariant results (Bril et al. 2010; Nonaka et al. 2010; Nonaka and Bril 2014; Nonaka 2011, 2013). This construct in particular highlights the importance of coordinating mechanisms like manuovisual and visuospatial integration.

As is common among primates, of all their senses, humans rely the most on haptic perception, active touch through the hand, and vision for their environmental interactions (Figure 1). As "one of the main perturbatory channels through which the world touches us, [the hand] has a great deal to do with how this world is perceived and classified" (Malafouris 2013:60). Haptic perception integrates sensory cues from what the fingers touch with internal 
sensory and positional cues about what the fingers feel and do (Hatwell et al. 2003). Its sensory dimensions are simultaneously internal and external, bipolarity that humans exploit (apparently, uniquely among animal species) in exploring their environments and making and using material culture (Mattens 2013). While sensing what objects are like by touching them undoubtedly supports the ability to explore and manipulate materiality, feeling what touches the body may be similarly important in helping broker interactions between human psychological abilities and the objects of material environments and cultures (Mattens 2013).

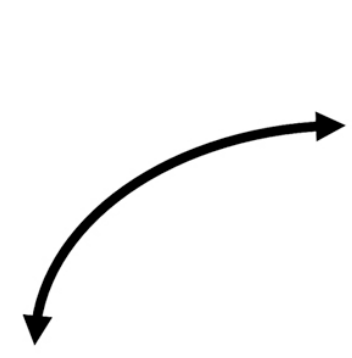

OUTER
SPACE
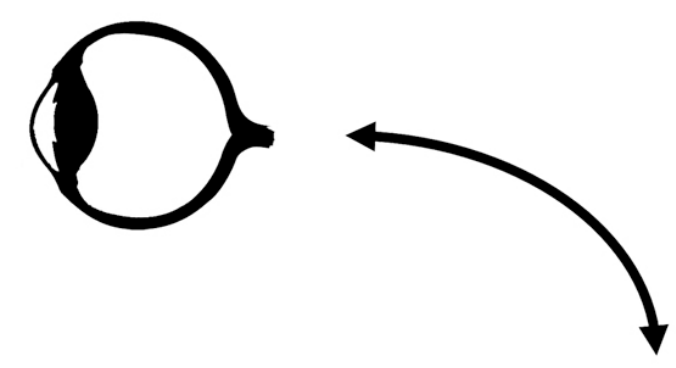

\section{INNER SPACE}

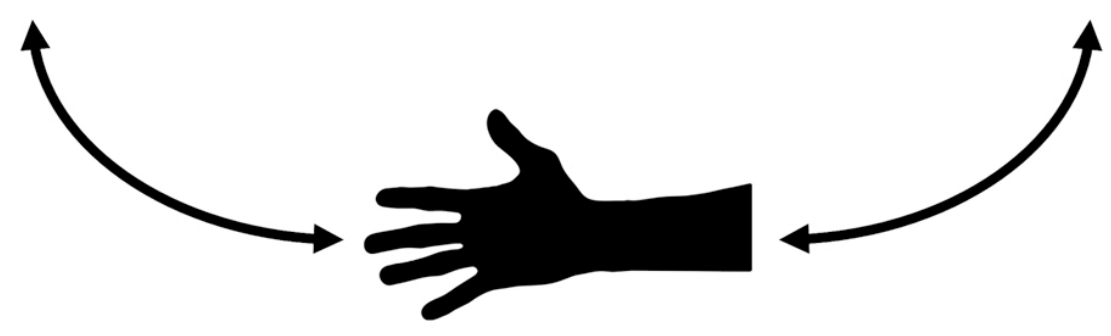

Figure 1. Although the entire body operates as an active interface between the brain and the environment, the eye and the hand in primates represent the two main ports of information flow and management.

Haptic perception provides a relatively limited perceptual field-only what the fingers can touch - that demands the fingers explore to gain sufficient information; the need to explore, coupled with high finger mobility and control, creates in touch a degree of alterability and intentionality that other senses lack (Hatwell 2003; Helbig and Ernst 2008). Vision, in contrast, provides a wide perceptual field easily explored through saccades and attentional shifts, enabling visual acquisition of more information in less time than is possible through touch, especially for informational domains where touch is particularly limited (e.g. spatiality, shape, etc.). Proficiency in interacting with an environment thus depends upon the abilities to gain pertinent sensory information, particularly about the locations and properties of manipulated objects; coordinate the inputs of multiple sensory modalities, most importantly touch and vision; and exercise fine control over and coordination of motor movements, especially for the hands and eyes (Gallagher 2013; Tallis 2003, 2011). 
In the enactivist paradigm, change in interacting neural, behavioral, and material resources cannot easily be isolated to the individual cognitive components. That is, brain function and form do not change on their own, but rather, through and because of interactivity with behavioral-material stimuli, and vice versa. Further, the constant state of change in human becoming (i.e. the ongoing and unfinished evolutionary process of becoming human) relates to both the capacity for change (plasticity) and the interactivity among the components of cognition. This is metaplasticity, "the enactive constitutive intertwining between neural and cultural plasticity" (Malafouris 2010a). The term brain-artefact interface (BAI) has been used to recognize that "the functional structure and anatomy of the human brain is a dynamic construct remodeled in detail by behaviourally important experiences which are mediated, and often constituted, by the use of material objects and cultural artefacts which for that reason should be seen as continuous integral parts of the human cognitive architecture" (Malafouris 2008:404, italics original).

BAIs are "technological mediations (material structures, processes, objects or other socio-material apparatuses and practices) that enable the configuration of a dynamic alignment or tuning between neural and cultural plasticity" (Malafouris 2010b:265). Crucially, BAIs in the form of material structures provide "temporal anchoring and binding" that helps bridge the vastly different timescales on which neural and cultural resources operate (Malafouris 2010b:266). While psychological responses and ontogenetic development take milliseconds to decades, materiality spans centuries to millennia or longer.

The idea that materiality not only reflects but also influences and changes brain function and form, especially over cultural and evolutionary spans of time, is perhaps not intuitive. Yet many species use or modify their behaviors and aspects of their environments in ways that can be considered tool use and culture (Laland and Janik 2006), and it is well accepted that physical anatomy and material environments influence behavior and psychological processing in most (if not all) species. Certainly, embodiment with pentadactyl limbs has strongly canalized the organization of number systems in cycles of 10 (decimal, the world's most prevalent numerical base), 5 (quinary), and 20 (vigesimal) (Comrie 2013; Menninger 1992), and manuovisual adeptness has been a primary enabler in allowing humans to use materiality to decompose tasks, collaboratively problem-solve, and-perhaps the most uniquely - accumulate cognitive effort that has been distributed over space and time (Beer 2003; Hutchins 1995).

Human manipulation of materiality into novel forms can also create new behavioral and psychological stimuli with the potential to change these cognitive components. Such interactivity opens up multiple cascading possibilities for further changes to the cognitive system, and over time may yield the kind of anatomical and behavioral differences that can be detected in the archaeological and fossil records. Examples include the change in spatial competence inferred from increasing symmetry in the shape of stone tools (Wynn 1989), as well as the intensification of the ability to incorporate materiality as a component of cognition inferred by comparing morphological change in brains and bodies in early $H$. sapiens and Neanderthals (Overmann 2015).

Given the importance of visuospatial integration to human becoming, a multidisciplinary approach is required to investigate the development of complexity in systemic behaviors arising from the interaction of brains (paleoneurology), bodies 
(paleontology), and materiality (archaeology). In this paper, we introduce three main sources of evidence for making inferences about visuospatial functions in extinct populations: neuroanatomy, technology, and ecology.

\section{Evolving parietal lobes}

The first source of evidence for investigating visuospatial functions in extinct species comes from their cranial remains: morphological changes in the neuroanatomical regions involved in visuospatial integration, particularly the parietal lobes. This region of the brain has been a particular research focus in the last decades (Mountcastle 1995; Zilles and PalomeroGallagher 2001). Initial interest examined general parietal functions like visuospatial integration and attention (Andersen and Buneo 2002; Andersen et al. 1997; Rushworth et al. 2001; Wardak et al. 2005), while more recently the analysis of the parietal cortex has been expanded to include more specialized processes like numbering (Ansari 2008; Cantlon et al. 2006). Studying the functions of the parietal lobes is challenging, for several reasons. First, as previously mentioned, relatively little attention has been given to them until fairly recently because of the perception that visuospatial functions were only associated with simple, mechanical behaviors. Second, these regions are involved in many different functions, with consequent operational difficulties in the design of reliable and comprehensive experimental paradigms. A third limit comes from the anatomy of these areas. The parietal elements primary involved in visuospatial management are the intraparietal sulcus and precuneus, both of which lie hidden in the depths of the cerebral volume (Ebeling and Steinmetz 1995). Such internal folds are more difficult to analyze in extant brains because of their secluded position within the brain mass. Dealing with fossils species, paleoneurology largely concerns the morphological evaluation of the outer surface, and changes of the inner cortical areas can be only partially evaluated. Their position also restricted those traditional approaches associating brain anatomy and behavior after physical or vascular lesions. In fact, individuals experiencing injuries or pathologies in deep cortical regions are less likely to survive, relative to individuals injured in more superficial brain districts. As a result, there are less data available to correlate regional damage with functional impairment. Finally, these regions are phylogenetically diverse. Differences among primate species are often so apparent that direct comparisons between corresponding areas are not easily established. As a result, parietal homologies between humans and non-human primates have yet to be reliably established.

In humans, intraparietal sulcus is larger and more complex than in other primates (Grefkes and Fink 2005; Orban et al. 2006; Vanduffel et al. 2002). Its areas are critical for eye-hand coordination (Battaglia-Mayer et al. 2006; Orban and Caruana 2014; Sakata et al. 1997). As might be expected, the intraparietal sulcus has been directly implicated in toolmaking (Stout and Chaminade 2007; Orban and Caruana 2014; Stout et al. 2015). The precuneus is a major functional and structural brain node, and it is involved in a wide array of behavioral processes. The precuneus appears to support two primary functions: it is a crucial node for visuospatial integration and a main connectivity hub for brain networks. As visuospatial node, it coordinates external and internal information to bridge the somatosensory cortex with vision and egocentric memory (Cavanna and Trimble 2006; Marguelis et al. 2009; Zhang and $\mathrm{Li}$ 2012). The precuneus has been implicated in generating self-centered mental representations, as well as coordinating spatial, chronological, and 
social information (Freton et al. 2014; Land 2014; Peer et al. 2015). It is particularly connected with the frontal cortex, as a key element of a highly integrated fronto-parietal system (Caminiti et al. 2015; Jung and Haier 2007). It is part of a network found only in humans thus far that joins the frontal and upper parietal areas and which is thought to be essential to the shift from emulation (reproducing results, a primate ability) to imitation (reproducing processes, a human ability) (Hecht et al. 2013). Its upper areas are strongly involved in visuospatial managements, through direct connections with the intraparietal sulcus and with the supramarginal gyrus, while its lower areas are more integrated with a central hub of the Default-Mode Network (Bzdok et al., 2015; Utevsky et al. 2014), a system that coordinates resting (or default) neural activity in absence of specific cognitive tasks (Buckner et al. 2008; Hagmann et al. 2008; Meunier et al. 2010). Interestingly, the precuneus is also particularly active in metabolic terms (Cavanna and Trimble 2006; Sotero and IturriaMedina 2011), suggesting that its evolutionary changes may have been associated with thermal constraints due to the brain's energy consumption and heat release (Bruner et al. 2014a). Interestingly, Alzheimer's disease, a pathology characterized by a combination of features found only in our species, is associated with early metabolic impairments in these deep parietal regions (Zhang and Li 2012; Bruner and Jacobs 2013).

The impression that visuospatial integration supports simple, mechanical behaviors has been significantly challenged by recent experimental evidence of neural change associated with interactions between body and tools (Iriki and Taoka 2012; Maravita and Iriki 2004). Tools induce different cognitive and neural responses when positioned outside the range of the body (extra-personal space) or when positioned within the range of physical interaction (peri-personal space). Most importantly, tools are incorporated into the body schemata when they are touched or handled: the brain interprets an object that is handled as an extension of the body. The classic example of this effect is the blind man's stick, whose neural incorporation extends tactile perception to the tip (Malafouris 2008). In this sense, objects function as "extra-neural" elements of the cognitive system, with the deep parietal areas serving to integrate internal neural processes with external environment features and managing the body's sensorimotor capacities as the interface between the two (Figure 2).

Although the whole body may act as an active interface, in primates the eye and the hand represent the main "ports" through which information is directed inward and outward, through an active integration of sensorimotor signals. In this process, the hand is not merely a biomechanical structure but also functions as a sensory device associated with neural feedback mechanisms that enables it to perform as an active biological interface, and not a mere passive anatomical component (Ingber 2008; Turvey and Carello 2011). It is interesting to note that handling processes (e.g. grasping objects, manipulating tools, and recognizing characteristics like size, texture, and quantity) are functionally subserved by distinct neural and cognitive mechanisms (Goodale et al. 1994). Therefore, "producing a tool” may rely on different processes than "using a tool" does, which in turn may only partially correspond to "feeling a tool" in terms of perception. Evolutionary changes in these processes have likely been complementary, but not necessarily identical or always integrated. 


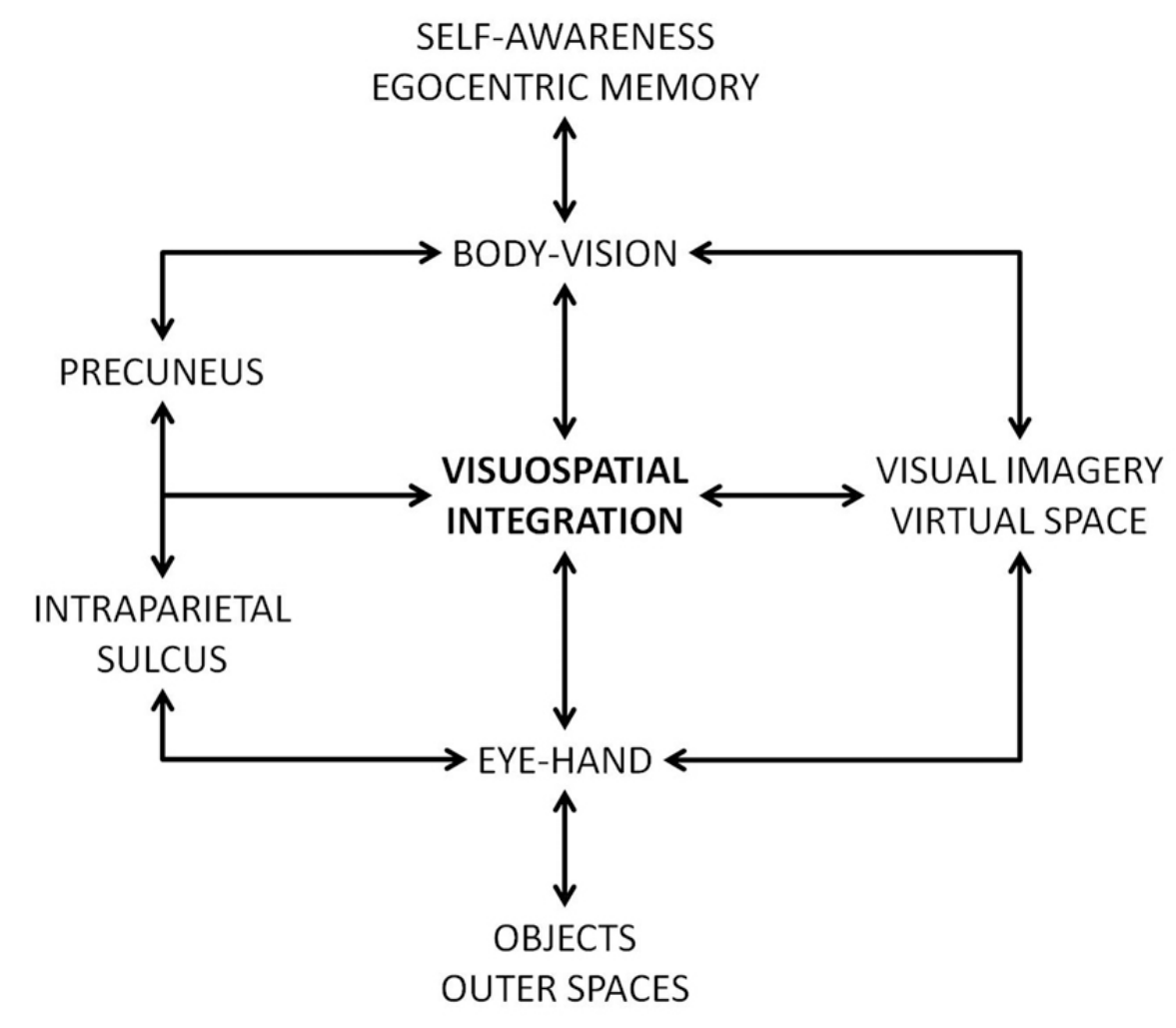

Figure 2. The upper and medial parietal areas (precuneus and intraparietal sulcus) are crucial for processes of visuospatial integration, generating a bridge between embodied experience and cognition.

Coordinating the outer and inner worlds is a main target in terms of ecology and adaptations, and it largely relies on visuospatial issues. The outer world is the environment and, for humans, environment means also culture, including technology. Tools enhance and influence our sensory and computational capacities, constrain and channel reasoning and decision-making processes, store information, and activate, catalyze, or induce responses in specific neural circuits. It is reasonable to believe that, among human populations, the less technological complexity the more the natural environment may be relevant in cultural terms. Conversely, the natural environment may lose importance as technology becomes a dominant mode of outer extension.

The inner world is the neural system, its organic extensions (somatosensory elements), and its intrinsic codes and representations. As previously mentioned, brains have historically been thought to recreate the external world internally, with the word representation used to describe the resultant stable and environment-independent simulations. However, there is no reason to think that representations are necessarily the result of disembodied processes (Prinz and Barsalou 2000). The current debate on this issue may be largely a matter of terminology, probably because the exact nature of such 
"representation” is not clear at all in biological or neurobiological terms. Physiologically, a representation may simply be a neural map that encodes sensory information (e.g. gained through visual means). Such mapping is not necessarily complete and self-sufficient, as it may rely on external components engaged through interactivity. And it is not necessarily stable, since it may depend on constant inputs and feedbacks from the body and the environment. As a matter of fact, humans generally think in images and language. Images need a virtual space in which we can perform simulations and mental experiments. Such "imaged space," whatever its actual biological background or neural substrate, is visuospatial in nature, largely self-centered, and based on spatial and chronological managements crucially coordinated by the precuneus (visual imagery; see Fletcher et al. 1995), that represent a functional bridge between body and vision. Interestingly, also regarding language, hypotheses concerning its evolution have associated its mechanisms with praxis, gesture, and movement (Binkofski and Buccino 2004; Buccino et al. 2005; Jirak et al. 2010; Marino et al. 2012), highlighting an additional importance of visuospatial managements.

Parietal growth and development have been associated with specific genetic factors (Chen et al. 2012), but they are also sensitive to training and behavioral effects (Hihara et al. 2006; Iriki and Taoka 2012; Quallo et al. 2009). Therefore, observed anatomical, physiological, or behavioral differences between individuals or groups (species or populations) can be primarily due to specific genetic characters, or else be the secondary effects of cultural training. The integration between biological and cultural factors may also promote hybrid evolutionary processes. For example, at the end of the nineteenth century James Baldwin hypothesized that learned behaviors can change the selective environment, channeling the following genetic selection towards culture-based directions (Baldwin 1896; Crispo 2007; Sznajder et al. 2012). In other words, the "Baldwin effect" holds that "under some conditions, learned behaviors can affect the direction and rate of evolutionary change by natural selection" (Depew 2003:3). Taking into account all these possibilities, evolutionary changes of the parietal functions in general and visuospatial capacities in particular can have been influenced by genetic, epigenetic, environmental, and cultural factors, and the extent to which each of these may have contributed to observed neural and behavioral differences between humans and non-human primates is currently unclear.

Paleoneurological analyses have provided compelling evidence of evolutionary change in parietal morphology. Though paleoanthropologists have long observed parietal anatomical differences and variations in early hominids and between extant and archaic humans (Dart 1925; Holloway 1981; Weidenreich 1941), quantitative data on evolutionary changes in parietal shape, size, and volume were not available until the last decade (Figure $3)$. Shape analyses suggest that the globularity of the modern human head is primarily related to an enlargement of the parietal bones (Bruner et al. 2004, 2011) that is in turn associated with an expansion of parietal lobes (Bruner 2004; Bruner et al. 2003). During development, this globularization takes place in an early stage present only in modern humans, and absent in Neanderthals and extant apes (Gunz et al. 2010; Neubauer et al. 2009, 2010; Scott et al. 2014). Most studies suggest that this stage occurs after birth, although there is the possibility that it takes place during gestation (Ponce de León et al. 2016).

The pattern of parietal expansion observed in modern humans is comparable to the pattern of enlargement of the precuneus observed in extant individuals, which is a major 
source of intra-specific variation in human brains (Bruner et al. 2014a, 2014b, 2015). This same pattern also represents a key difference between human and chimpanzee midsagittal brain morphology, because of a noticeable enlargement of the precuneus in our species (Bruner et al. 2016a). Interestingly, early modern humans may have lacked the parietal bulging that characterizes $H$. sapiens (Bruner and Pearson 2013), with some later specimens displaying an intermediate phenotype (Bruner et al. 2016b). This evidence is still based on very few individuals but, if confirmed, it separates the origin of the modern human lineage from the origin of the modern human brain form. This raises once more the question of whether such changes where gradual or discrete, and whether associated with genetic selection or environmental feedback (Bruner and Iriki 2016).

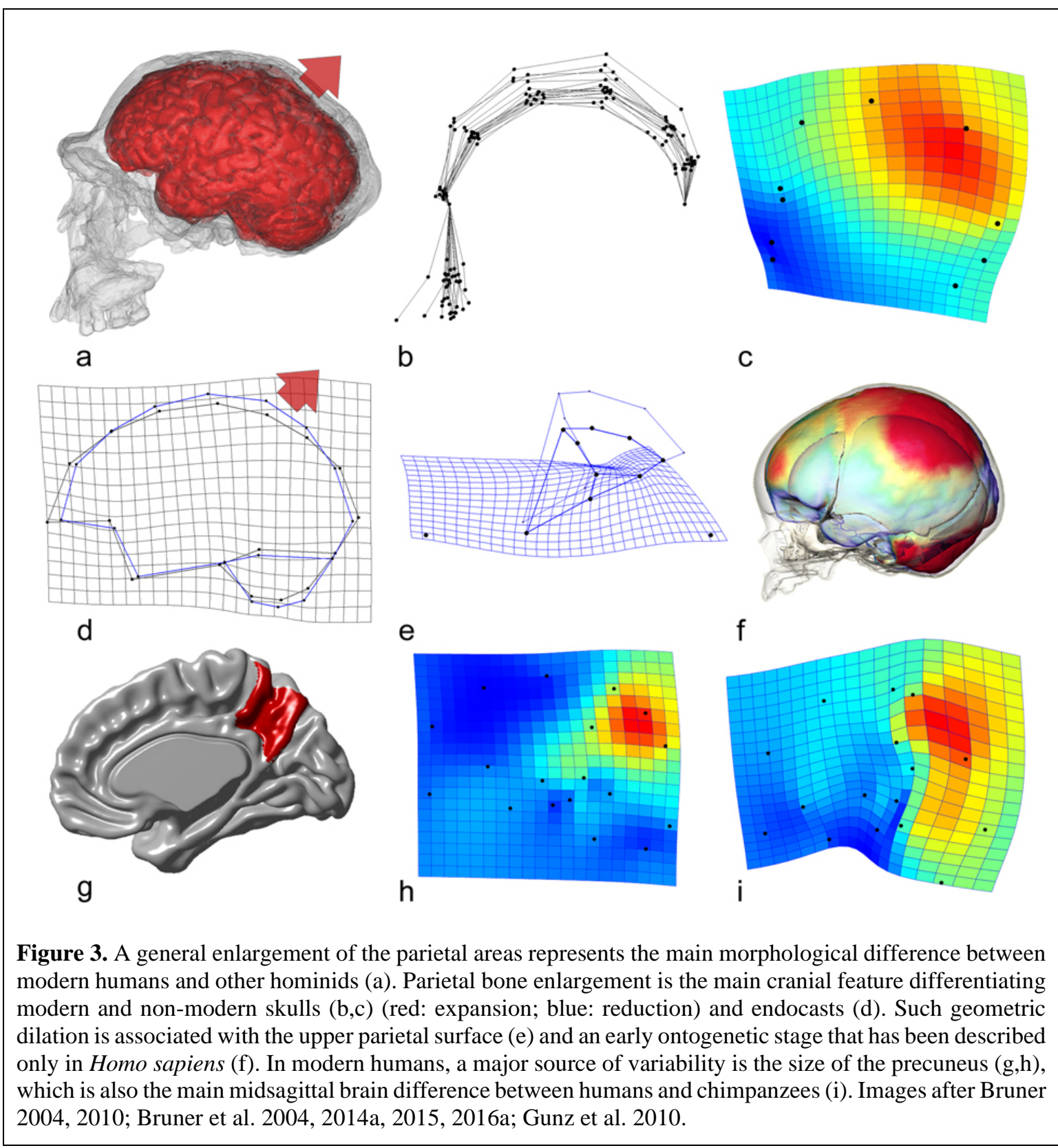


Apart from these gross neuroanatomical differences dealing with size and proportions, comparative analyses to date have failed to localize any specific structural or functional difference of the parietal cortex between humans and other primates (Caminiti et al. 2015; Caspers et al. 2011; Mars et al. 2011; Scheperjans et al. 2008). Also, its role in the Default-Mode Network seems comparable in different species (Barks et al. 2015; Rilling et al. 2007). All these results suggest that the general organization, connectivity, and processes of the parietal lobes are generally conserved. Therefore, we must evaluate whether differences in human parietal anatomy and functions have been a matter of new elements and mechanisms (i.e. derived characters), the reuse of primitive traits (exaptation), or simply a matter of the degree of expression of shared abilities (a plesiomorphic scheme enhanced in terms of capacity, possibly with threshold effects).

Although the parietal surface displays the most noticeable morphological change between modern and non-modern humans, other brain areas represent important nodes for functions associated with spatial behavior. For example, spatial mapping relies on medial temporal elements, and there is direct quantitative evidence of larger temporal lobes in our species compared to extant apes (Rilling 2006; Rilling and Seligman 2002). Actually, the temporal poles in extant humans are also more projected relative to those of other human species, at least when the morphology and position of the middle endocranial fossa are considered (Bastir et al. 2008).

In sum, we should consider three distinct issues: (1) $H$. sapiens is characterized by a unique morphological expansion of the parietal bones and lobes; (2) the intraparietal sulcus and the precuneus, implicated in a number of critical functions related to visuospatial behaviors, are localized in those same areas, and represent a relevant source of variability both within and between extant and extinct species; and (3) the possibility that these two facts are somehow related. It remains to be established to what extent these morphological changes are the result of genetic, selection, and environmental influences. In all cases, taking into consideration the conspicuous parietal changes and the importance of culture and tooling in our species, the exploration and analysis of visuospatial functions merits attention in evolutionary anthropology and behavioral neuroanatomy.

\section{Tools and tooling: changing perspectives}

The second line of evidence for investigating visuospatial functions in past populations comes from their tools and the elements and processes associated with tooling. Extant humans evolved from ancestors who had the ability to make and use tools, and material culture plays a major role in current evolutionary theories: behaviors with materiality are an essential part of hominid evolution, bridging adaptive responses, natural selection, and cognitive capacities (Foley and Lahr 2003). Technology and tooling are thus hallmarks of human evolution, and there is no doubt that we provide the most complex example of technology-based species. Because stone was an essential resource for Paleolithic groups, and because it has the property to persist sometimes over million years, we largely rely on it to make inferences about the behavior of extinct prehistoric populations.

The interaction between the brain and body is a focal point for thinking about the change in tool form and use over time, and about the interaction between hominids and artefacts. Because they are made of a material whose durability makes them more likely to 
preserve, lithics are the most common archaeological objects. Lithics are often the only vestige of past behavior for an extinct population or species. The stone tool archaeological record also covers an enormous span of time: from over 3 Mya to virtually the present (Figure 4). Thus the interaction of brains and tools through the interface of hands and eyes fundamentally underpins and pervades the evolutionary history of our species. As Robert Foley (1987:381) noted, "covariation between hominid fossil morphology and artefact variability is an essential starting-point for understanding the evolution of human behavior," with visuospatial integration offering a behavioral bridge between evolutionary neuroscience and prehistoric archaeology.

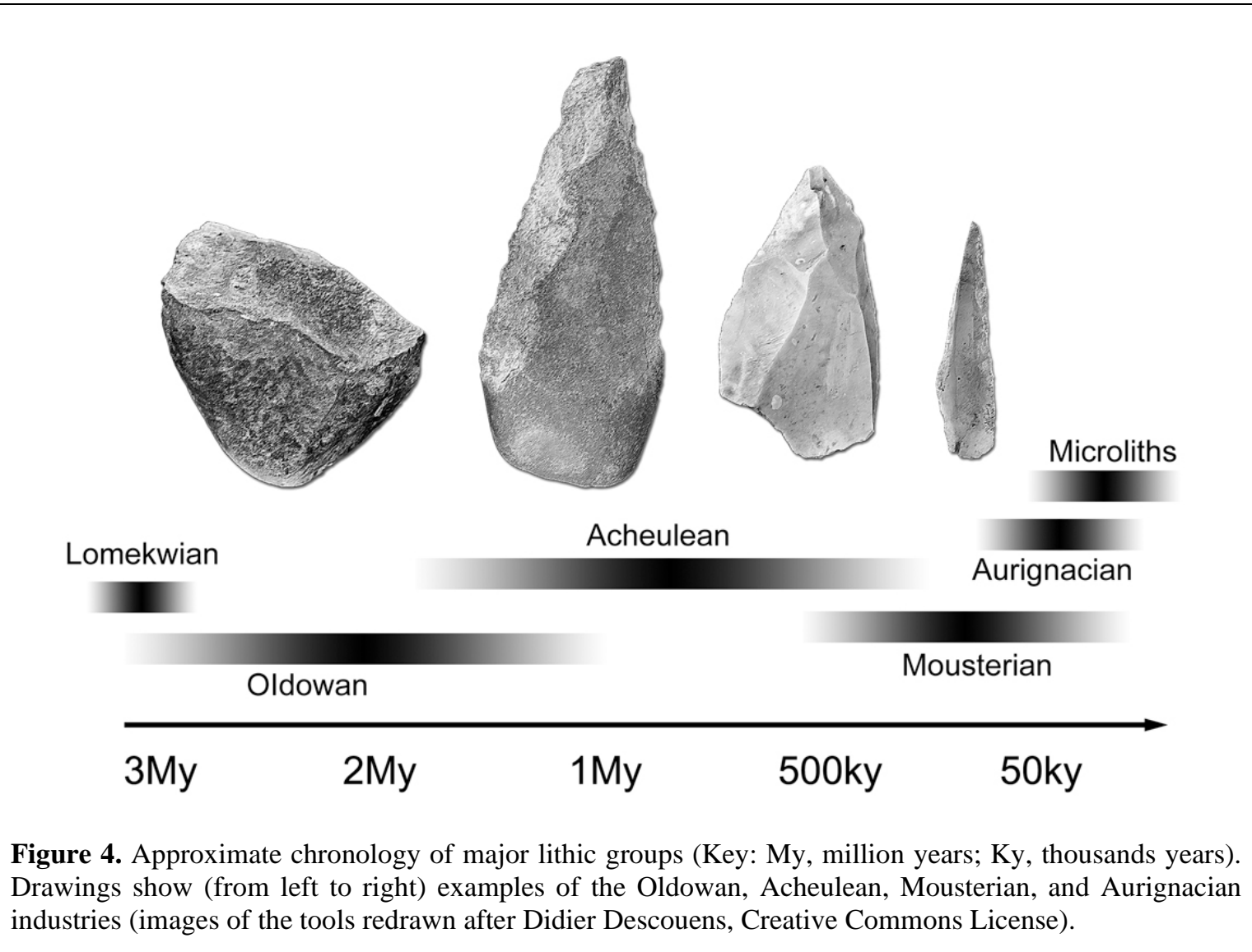

An attempt to account for the evolution of tooling is the "mode” model (Clark 1969), based on the relation between the core and the product. Mode 1 represents the simplest flaking technology, linked to the Oldowan industry. Mode 2 corresponds to Acheulean (shaping and producing larger flakes), Mode 3 to the prepared core technologies of the Middle Palaeolithic sensu lato. Finally, modes 4 and 5 are associated with, respectively, the production of blades and microliths. The sympatric coexistence of different hominids species sharing the same technocomplex, challenges the one-to-one relations between particular species and the cultural innovation represented by specific lithic technologies. Nonetheless, the mode division enables an initial tentative identification of distinct cognitive schemes with different evolutionary and adaptive implications. Most importantly, the model enables 
plausible associations between particular cognitive processes and the adaptive behaviors producing specific technocomplexes (Foley and Lahr 2003).

Considered in total, the rate of change in lithic technologies is relatively slow, with very long periods without significant innovations followed by apparent changes associated with novel patterns. These innovations often seem the outcome of the combination of technical ideas already existing and other generated independently (Beaune et al. 2009), and in this sense they could be considered a form of exaptation (sensu Gould and Vrba 1982).

The shift from tool-assisted to tool-dependent foraging, may signal an important cognitive change in hominds (Plummer 2004), moreover one that would have introduced culture ipso facto into their cognitive and neural niches (Iriki and Taoka 2012). During handling, tools are included in the "body schemata" of the brain, and thus are integrated within the neurological mechanisms that affect the way we move and we perceive the space around us (Maravita and Iriki 2004). The cognitive foundations connected with tool making are controversial, involving many distinct areas and processes (Stout and Chaminade 2007), and lithic studies have often been used to provide different order of understanding about hominid cognitive capacities, from mental imagery to plan specific knapping goals. However, exactly what that cognitive change may have consisted of has been difficult to define. Lithic production has been decomposed into sequences of steps and decisions (the chaîne opératoire approach) in attempts to understand, among other aspects, its cognitive underpinnings. For example, the concept of "method" in lithic studies implies the repeated organization of the knapping operation, traditionally linked to a "mental preparation" before the knapping itself (Tixier 1967). To illustrate this point, Pelegrin (2009) compared the knapping process to a game of chess, in which many preparatory steps are aimed at producing the conditions needed for the desired outcome. Such analyses have been used to argue for different understandings of hominid cognitive capacities, from mental imagery to planning specific knapping goals, and indeed, the full extent of the cognitive foundations of tool making remain unsettled, as they involve multiple, distinct brain regions and processes (Stout and Chaminade 2007). Further, cognitive models of lithic production have not fully incorporated yet insights like the neurological incorporation of tools, when they are handled, into mechanisms affecting movement and spatial perception (Maravita and Iriki 2004).

However, if we interpret mind as an integrated between brain, body, and environment, the analogy of a computer-like process performed by activity in the brain, becomes too reductive. If the body and the material culture are indeed implicit components of cognition, both hands and tools must be the target of a more comprehensive behavioral and cognitive analysis. Certainly, the hand/tool relationship is not unidimensional: the hand not only produces but also uses the tool, actions that may be managed by complementary but distinct cognitive processes. Hand and tool variation can be the result of reciprocal influence channeling their properties through information that is external to the brain and central nervous system but which is necessary to and implicit in the cognitive responses.

Managing relationships between brain, body, and tools, relies on visuospatial processes, and for this reason changes associated with the body and material culture can reveal underlying differences in visuospatial behavior, allowing inferences about change in visuospatial functions. Evidence of visuospatial behaviors can be direct or indirect. 
Direct information providing evidence of visuospatial behaviors can be obtained from tool manufacture and use and from hand morphology, as well as the integration of the two. Behaviours associated with tools and tooling can be investigated through experimental replication of prehistoric tools, observation of stone making in contemporary human groups, and observation of the tool-use in apes (Marzke and Shackley 1986; Toth et al. 1993a; Toth et al. 1993b; Schick et al. 1999).

Following Napier (1956), hand morphology and tool evolution have been major topics in anthropology and functional anatomy (Marzke 2013; Marzke and Marzke 2000). Bipedalism, which freed the hands and upper limbs from locomotion, set the basic conditions for the evolutionary emergence of tooling, with locomotor and manipulation abilities evolving in mosaic fashion rather than following a gradual trend (Kivell et al. 2011). Morphological advantages of the human hand with respect to apes include precision grip (Napier 1956) and "squeeze" grip (Marzke 1997), facilitating the ability to handle a stone hammer. Precision movements of the wrist are also considered significant. However, the presence of pad-to-pad precision grasping in the genus Orrorin suggests that some of the basic features of the humanlike hand may be plesiomorphic for hominids (Almecija et al. 2015).

Grasping patterns can be classified in terms of hand-tool interaction (Feix et al. 2015), and the hand's functional morphology can be analyzed within a specific archaeological context (Williams et al. 2012). This physical interaction requires a proper integration between the motor system, the properties of the object, and the action itself (Vankof and Kokinov 2013). These kinds of studies can be modeled by using different quantitative (Liu and Zhan 2013) and comparative (Pouydebat et al. 2009; Rolian et al. 2011) approaches, and their applications range from evolution to robotics (Kappassov et al. 2015). Interestingly, Neanderthals and coeval modern humans in the Levant may have differed in their hand anatomy while using similar technology (Churchill 2001; Niewoehner 2001).

Although precision and hand dexterity are crucial for tooling, nonetheless they are not a necessary requisite for cognitive extension. A machine (e.g., a mechanic arm) can be far more precise than a human hand, but its close program prevents any cognitive mechanisms. Therefore, we should consider whether precision is a cause or else an evolutionary consequence of prostethic capacity. As previously mentioned, beyond purely mechanical issues, the body responds to tactile stimulations according to a system of tensions and stresses in which the process of converting mechanical signals into biochemical responses is synchronized with perceptive feedback, and hence directly with behavioral and cognitive experience (Ingber 2008; Turvey and Carello 2011). How such relationships might influence tool manufacture and use, however, remains to be investigated.

Indirect information of visuospatial behaviors can be obtained from remains that imply specific activities. For example, Neanderthals and their common ancestor with $H$. sapiens displayed frequent and multiple scratches on their anterior teeth, suggesting they used their mouths as "third hand" to a larger extent than found in contemporary $H$. sapiens or any extant human population (Lozano et al. 2008). Because Neanderthals relied more extensively on the mouth for handling and also lacked the upper parietal expansion characteristic of contemporary H. sapiens, Bruner and Lozano (2014, 2015) have hypothesized that Neanderthals may have lacked specialized visuospatial system and embodying capacity possessed by modern humans. The hypothesis can be evaluated through 
expanded analyses of the paleontological and archaeological information, investigation of mouth-hand exploratory behaviors during ontogenetic development, examination of ethnographic parallels in living populations of hunter gatherers, and functional analyses of mouth-hand integrative relationships.

Since making stone tools is a social behavior (i.e. learned and reproduced through social processes of observation, reproduction, practice, and perhaps language), it depends on cognitive capacities, processes, and skills that exceed those of the individual (e.g. Lycett 2011; Tostevin 2013). Even expedient tooling implies accumulated knowledge, social transmission and learning, and an understanding of the mineral environment, factors that become more critical as tool complexity increases. These features suggest that tooling as a cognitive issue is intimately connected with the social environment. Since tools and social systems can exert reciprocal influences, the archaeological record may be able to detect and relate change in both components.

About tooling and social structure, handling capacities have often been associated with language (Binkofski and Buccino 2004; Leroi-Gourhan 1964). In extant populations, language appears to play an active role in learning and teaching manufacturing techniques (Uomini and Meyer 2013). As mentioned, it has been recently hypothesized that language comprehension may be strongly embodied, requiring a response of the motor system to code words and actions (Corballis 1999; Gentilucci and Corballis 2006; also see Buccino et al. 2005; Marino et al. 2012). This possibility suggests that we should consider a scenario involving the hand-tool circuit, language, and the social system, with visuospatial capacity integrating and managing the relations between brain, body, and environment.

\section{Evolving spaces}

A third way to investigate visuospatial functions is to examine the spatial behavior of past human populations. Complex patterns of landscape use can be reconstructed through the distribution of archaeological sites, archaeozoological evidence of seasonal patterns of occupation, and evidence for the circulation of raw materials and finished artifacts which act as proxies for human mobility. Patterns of mobility and the maintenance of social networks within settlement systems are activities that rely upon spatial cognition and wayfinding ability.

Wayfinding, the ability to plan and carry out navigation tasks, implies an ability to create abstract mental representations of space. Two distinct representations of the world are linked by a process of translation: first, an egocentric representation that involves "transient action-oriented egocentric self-object associations” and second, an allocentric representation with a "more enduring ... object-object or environment-object association" (Burgess 2006:555). The allocentric representation implies the adoption of a "survey" perspective, also known as a "bird's-eye view." The translation process, known as spatial updating, allows agents to translate their current position onto an enduring, allocentric representation of space which functions as a "cognitive map," an internal representation of the world that acts as a framework for planning further action and is used metaphorically during memory recall (Golledge 2003:30). 
The hippocampal formation plays an important role in generating spatial representations Roche et al. 2005; Nadel and Hardt 2004; Spiers and Maguire 2006; Maguire et al. 1999, 2000, 2003; Etienne and Jeffery 2004). The parahyppocampal and perirhinnal cortices are the main links between the hippocampal formation and the sensory systems (Roche et al. 2005) thus playing a critical role in path integration (Whitlock et al. 2008). The posterior parietal cortex integrates the sensory and motor systems and plays a key role in planning specific actions and enabling goal-directed movement (Spiers and Maguire 2006; Whitlock et al. 2008). Together, these different regions of the brain contribute to the creation of the spatial representations used during wayfinding.

Spatial representations imply the motivated and deliberate encoding of environmental information, they are built up from direct experience of the world (or from external aids, such as maps) and constantly updated as an agent moves through space. There are two distinct strategies used during this process: path integration and landmark recognition (Foo et al. 2005). Path integration (aka "dead reckoning”) is the ability to update one's position on the allocentric representation using inertial signals processed by the vestibular system (Etienne and Jeffery 2004) and Euclidian geometrics (bearings and path lengths, or distance and direction). Landmark recognition (the ability to select relevant features of the landscape and remember their position) is used when adopting an egocentric perspective and also comes into play during the process of translating between spatial perspectives to create an integrated cognitive map. Path integration and landmark recognition are not mutually exclusive processes since external, location-based cues can be combined with inertial signals during wayfinding; humans (and other animals) use these wayfinding strategies contextually and individual preferences are also evident.

Individual differences in spatial ability may reflect differences in cognitive style or underlying differences in cognitive ability (Bosco et al. 2004; Burke et al. 2012; Saucier et al. 2002). A link between spatial ability and navigation outcomes is apparent, and tests of mental rotation, visualization, and memory are good predictors of successful wayfinding (Allen 1999). A training effect has also been demonstrated (Feng et al. 2007), with structural changes to the brain observable in highly trained individuals (e.g. the well-known case study involving London cab drivers; see Maguire et al. 2000). In other words, the brain is capable of responding physiologically to the demands of regularly performed spatial tasks.

The archaeological record suggests that modern humans developed complex, spatially extensive social networks during the course of the Middle Stone Age (280-50 Kya) and developed the tools and social conventions necessary to maintain them (d'Errico et al. 2003, 2005; Gamble 1998; Wadley 2001). The symbolic use of material culture to define social relationships is considered typical of modern human behaviour and is thought to emerge during the Middle Stone Age (Henshilwood and Marean 2003; Wadley 2001). The ethnographic record shows that the reciprocal exchange of artefacts functions to maintain social networks (Weissner 1984) and the manufacture of items of personal adornment (e.g., beads, pendants) during the Palaeolithic could signal the symbolic use of material culture as a marker of social identity, used to cement social relationships and maintain social networks. Supporting evidence for the development of more spatially extensive networks during the Upper Palaeolithic of Europe includes the long-distance circulation of "exotic" raw materials (Feblot-Augustin 1993; Feblot-Augustin 1997). 
Hunter-gatherers use social networks to collect information about resource availability (Whallon 2006; Whallon et al. 2011). They also act as a form of "social safety net" since the social obligations engendered through reciprocal exchanges between members of a network can be exploited in times of resource stress (Kelly 2013; Cashdan 1985; Weissner 1982; Smith 1988). Keeping track of dynamic, spatially extensive social networks would have placed additional cognitive demands on the brain and may have enhanced spatial ability, as well as contributing to the dispersibility of modern human populations (Burke 2012).

While the development of complex, spatially extensive social networks might have selected for enhanced spatial cognition, the reverse may also be true. That is, it is possible that structural changes in the human brain, such as the enlargement of the parietal and temporal regions described earlier, enhanced spatial cognition and facilitated the development of dynamic, long-distance social networks. Similarly, it has been hypothesized that enhanced working memory (associated with neurological changes in the frontal lobes) is a key aspect of modern human cognition that promoted planning skills and the development of managed foraging systems, albeit at a much later date (Wynn and Coolidge 2003). Whether or not structural changes to the brain translated into more efficient wayfinding remains to be seen, but it is possible that the role of the parietal and temporal regions in coordinating goal-directed movement and encoding environmental signals was enhanced with implications for path integration and wayfinding ability. This would have permitted modern humans to travel and explore new regions with greater ease, facilitating the development of modern human social networks and enhancing the ability of human groups to disperse widely - a phenomenon observable in the archaeological record of the Late Pleistocene.

\section{Spaces and minds: visuospatial cognition and social aspects}

If humans experienced a change or a specialization for extending their cognitive capacities beyond the brain, then visuospatial functions may well represent a focal point for future investigation in anthropology and archaeology (Bruner and Iriki 2016). The visuospatial system can be partially investigated in paleoanthropology taking into account evolutionary changes in the anatomical elements involved in visuospatial functions (Bruner et al. 2016c). Visuospatial integration can be studied in physical and cultural anthropology by using the comparative approach, and by testing differences in visuospatial behaviors associated with specific tools or environments. Following a traditional approach in human ethology (EiblEibesfeldt 1989), universal visuospatial behaviors (i.e., those considered to be independent of culture) can reveal shared human capacities channeling the cognitive experience. In terms of neurobiology, visuospatial processes can be investigated in extant human populations through functional imaging and psychometrics. Taking into account that modern humans rely on dental manipulation less than other hominids did, it may be interesting to evaluate in greater detail the neurological mechanisms involved in the ontogenetic transition from mouth-based to hand-based exploration in infants (Haggard and De Boer 2014; Rochat 1989, 1993). Regarding cognitive performance, preliminary psychometric analyses have thus far not revealed correlations between precuneus dimensions and standard visuospatial cognitive tests (Bruner et al. 2015). Nonetheless, there is a possibility that psychometric evaluation of parietal functions should be based on batteries tailored to integrative aspects of visuospatial 
cognition or embodiment capacities. In archaeology, visuospatial functions can be investigated by interpreting visuospatial behaviors, following the principle of cognitive archaeology (Coolidge and Wynn 2005, 2009; Coolidge et al. 2015). Tool forms and structures, as well as activities like hunting, visual arts, and land use, can provide direct and indirect evidence of specific behaviors, which may yield variables and parameters that can quantitatively support specific cognitive hypotheses.

We are now realizing that the body and material culture may play a major role within human cognition (Byrge et al. 2014; Haggard 2005). This relationship may become even more complex when different bodies interact (i.e. when dealing with interpersonal and social relationships). In fact, the spatial and chronological perception associated with the self and others strongly influences how social structures are perceived (Hills et al. 2015; Maister et al. 2015). The individual self may think of sociality in terms of a "social space" with spatial and chronological properties. Through autonoetic perspectives based on the recognition of the self in time and space, we manage our representations of relationships between persons, environments, and objects (Peer et al. 2015). Probably not by chance, there is a special link in primates between brain dimension, social structure, and touch (Dunbar 1998, 2008): brain size appears proportional to group size, which in turn appears proportional to grooming activity. These three factors exert a reciprocal influence in the sense that they can promote or constrain each other. Whatever the actual causes and effects, brain size, social structure, and physical contact are profoundly associated. Interestingly, the relationship between touch and group behaviors is mediated by endorphins, representing an organic bridge between touch, brain, and society (Dunbar 2010; Machin and Dunbar 2011; Suvilehto et al. 2015; Nummenmaa et al. 2016).

Because of the limited information available on the mechanisms of visuospatial integration and embodiment, the first priority will be to identify experimental paradigms that will enable the exploration of these functional networks, and the evaluation of specific cognitive hypotheses. Although many evolutionary processes cannot be tested, and although cognition cannot be directly investigated in fossil species, an effort must be done to assess the consistency of hypotheses in cognitive archaeology through quantitative comparisons and multidisciplinary convergence. The second priority is to look for evidence of possible change in the paleontological and archaeological records that relate to visuospatial behaviors. The evolutionary perspective can reveal gradual or discontinuous variations in the way we perceive and interact with the external environment, integrating and enhancing our neural processes with additional extended resources.

\section{Acknowledgments}

We are grateful to the many friends, colleagues, and students who helped us develop the topics introduced in this paper: Fred Coolidge, Tom Wynn, Lambros Malafouris, Duilio Garofoli, Eiluned Pearce, Atsushi Iriki, Jim Rilling, Annapaola Fedato, Roberto Colom, Manuel Martin-Loeches, Ralph Holloway, Marina Lozano, Joseba Rios Garaizar, and Sileshi Semaw. EB is supported by the Spanish Government (MINECO CGL2015-65387-C3-3P). EES is supported by a SIR (RBSI142SRD) grant from the MIUR (Italian Ministry of University and research). 


\section{References}

Allen GL (1999) Spatial abilities, cognitive maps, and wayfinding. Basis for individual differences in spatial cognition and behavior. In: Golledge RG (ed) Wayfinding behavior. Johns Hopkins University Press, Baltimore.

Almécija S, Wallace IJ, Judex S, Alba DM, Moyà-Solà S (2015) Comment on ape human-like hand use in Australopithecus africanus. Science 348:1101-1101.

Andersen RA, Buneo CA (2002) Intentional maps in posterior parietal cortex. Ann Rev Neurosci 25:189-220.

Andersen RA, Snyder LH, Bradley DC, Xing J (1997) Multimodal representation of space in the posterior parietal cortex and its use in planning movements. Ann Rev Neurosci 20:303-330.

Ansari D (2008) Effects of development and enculturation on number representation in the brain. Nat Rev Neurosci 9:278-291.

Baldwin MJ (1896) A new factor in evolution. Am Nat 30:441-451.

Barks SK, Parr LA, Rillin JL (2015) The default mode network in chimpanzees (Pan troglodytes) is similar to that of humans. Cerebr Cortex 25:538-544.

Bastir M, Rosas A, Lieberman DE, O’Higgins P (2008) Middle cranial fossa and the origin of modern humans. Anat Rec 291:130-140.

Battaglia-Mayer A, Archambault PS, Caminiti R (2006) The cortical network for eye-hand coordination and its relevance to understanding motor disorders of parietal patients. Neuropsychologia 44:2607-2620.

Beer RD (2003) The dynamics of active categorical perception in an evolved model agent. Adaptive Behav 11:209-243.

Binkofski F, Buccino G (2004) Motor functions of the Broca’s region. Brain Lang 89:362-369.

Bosco A, Longoni AM, Vecchi T (2004) Gender effects in spatial orientation: cognitive profiles and mental strategies. App Cogn Psychol 18:519-532.

Bril B, Rein R, Nonaka T, Wenban-Smith F, Dietrich G (2010) The role of expertise in tool use: skill differences in functional action adaptations to task constraints. J Exp Psych 36:825-839.

Bruner E (2004) Geometric morphometrics and paleoneurology: brain shape evolution in the genus Homo. J Hum Evol 47:279-303.

Bruner E (2010) Morphological differences in the parietal lobes within the human genus. Curr Anthropol 51:S77-S88.

Bruner E, Iriki A (2016) Extending mind, visuospatial integration, and the evolution of the parietal lobes in the human genus. Quat Int 405:98-110.

Bruner E, Jacobs HIL (2013) Alzheimer's disease: the downside of a highly evolved parietal lobe? J Alzheimer's Dis 35:227-240.

Bruner E, Lozano M (2014) Extended mind and visuo-spatial integration: three hands for the Neandertal lineage. J Anthropol Sci 92:273-280.

Bruner E, Lozano M (2015) Three hands: one year later. J Anthropol Sci 93:191-195.

Bruner E, Pearson O (2013) Neurocranial evolution in modern humans: the case of Jebel Irhoud 1. Anthropol Sci 121:31-41.

Bruner E, Manzi G, Arsuaga JL (2003) Encephalization and allometric trajectories in the genus Homo: Evidence from the Neandertal and modern lineages. Proc Natl Acad Sci USA 100:15335-15340.

Bruner E, Saracino B, Ricci F, Tafuri M, Passarello P, Manzi G (2004) Midsagittal cranial shape variation in the genus Homo by geometric morphometrics. Coll Anthropol 28:99-112.

Bruner E, de la Cuétara JM, Holloway R (2011) A bivariate approach to the variation of the parietal curvature in the genus Homo. Anat Rec 294:1548-1556. 
Bruner E, Rangel de Lázaro G, de la Cuétara JM, Martín-Loeches M, Colóm R, Jacobs HIL (2014a) Midsagittal brain variation and MRI shape analysis of the precuneus in adult individuals. J Anat 224:367-376.

Bruner E, de la Cuétara JM, Masters M, Amano H, Ogihara N (2014b) Functional craniology and brain evolution: from paleontology to biomedicine. Front Neuroanat 8:1-19.

Bruner E, Román FJ, de la Cuétara JM, Martín-Loeches M, Colóm R (2015) Cortical surface area and cortical thickness in the precuneus of adult humans. Neuroscience 286:345-352.

Bruner E, Preuss T, Chen X, Rilling J (2016a) Evidence for expansion of the precuneus in human evolution. Brain Struct Funct doi:10.1007/s00429-015-1172-y.

Bruner E, Amano H, Pereira-Pedro S, Ogihara N (2016b) The evolution of the parietal lobes in the genus Homo. In: Bruner E, Ogihara N, Tanabe H (eds) Digital endocasts. Springer, Tokyo (in press).

Bruner E, Lozano M, Lorenzo C. (2016c) Visuospatial integration and human evolution: the fossil evidence. J. Anthropol. Sci. 94: 81-97.

Buccino G, Riggio L, Melli G, Binkofski F, Gallese V, Rizzolatti G (2005) Listening to action-related sentences modulates the activity of the motor system: a combined TMS and behavioral study. Cogn Brain Res 24:355-363.

Buckner RL, Andrews-Hanna JR, Schacter DL (2008) The brain's default network. Ann New York Acad Sci 1124:1-38.

Burgess N (2006) Spatial memory: how egocentric and allocentric combine. Trends Cogn Sci 10:551-557.

Burke A (2012) Spatial abilities, cognition and the pattern of Neanderthal and modern human dispersals. Quat Int 247:230-235.

Burke A, Kandler A, Good D (2012) Women who know their place: sex-based differences in spatial abilities and their evolutionary significance. Hum Nat 23:133-148.

Byrge L, Sporns O, Smith LB (2014) Developmental process emerges from extended brain-body-behavior networks. Trends Cogn Sci18:395-403.

Bzdok D, Heeger A, Langner R, Laird AR, Fox PT, Palomero-Gallagher N, Vogt BA, Zilles K, Eickhoff SB (2015). Subspecialization in the human posterior medial cortex. NeuroImage 106:55-71.

Caminiti R, Innocenti GM, Battaglia-Mayer A (2015) Organization and evolution of parieto-frontal processing streams in macaque monkeys and humans. Neurosci Biobehav Rev 56:73-96.

Cantlon JF, Brannon EM, Carter EJ, Pelphrey KA (2006) Functional imaging of numerical processing in adults and 4-y-old children. PLoS Biol 4:844-854.

Cashdan EA (1985) Coping with risk: reciprocity among the Basarwa of Northern Botswana. Man (New Series) 20 (3):454-474.

Caspers S, Eickhoff SB, Rick T, von Kapri A, Kuhlen T, Huang R, Shah NJ, Zilles K (2011) Probabilistic fiber tract analysis of cytoarchitectonically defined human inferior parietal lobule areas reveals similarities to macaques. NeuroImage 58:362-380.

Cavanna AE, Trimble MR (2006) The precuneus: a review of its functional anatomy and behavioural correlates. Brain 129:564-583.

Chen CH, Gutierrez ED, Thompson W, Panizzon MS, Jernigan TL, Eyler LT, Fennema-Notestine C, Jak AJ, Neale MC, Franz CE, Lyons MJ, Grant MD, Fischl B, Seidman LJ, Tsuang MT, Kremen WS, Dale AM (2012) Hierarchical genetic organization of human cortical surface area. Science 335:1634-1636.

Churchill SE (2001) Hand morphology, manipulation, and tool use in Neandertals and early modern humans of the Near East. Proc Natl Aca Sci USA 98:2953-2955.

Clark A (1997) Being there: putting brain, body, and world together again. MIT, Cambridge. 
Clark A (2008) Supersizing the mind: embodiment, action, and cognitive extension. Oxford University Press, New York.

Clark A, Chalmers DJ (1998) The extended mind. Analysis 58:7-19.

Clarke JGD (1969) World prehistory: a new outline. Cambridge University Press, Cambridge.

Comrie B (2013) Numeral bases. In: Dryer MS, Haspelmath M (eds) The world atlas of language structures online. Max Planck Institute for Evolutionary Anthropology, Leipzig.

Coolidge FL, Wynn T (2005) Working memory, its executive functions, and the emergence of modern thinking. Camb Archaeol J 15:5-26.

Coolidge FL, Wynn T (2009) The rise of Homo sapiens: the evolution of modern thinking. Wiley-Blackwell, Oxford.

Coolidge FL, Wynn T, Overmann KA, Hicks JM (2015) Cognitive archaeology and the cognitive sciences. In: Bruner E (ed) Human paleoneurology. Springer, Cham.

Corballis MC (1999) The gestural origins of language: human language may have evolved from manual gestures, which survive today as a 'behavioral fossil' coupled to speech. Am Sci 87:138-145.

Crispo E (2007) The Baldwin Effect and genetic assimilation: revisiting two mechanisms of evolutionary change mediated by phenotypic plasticity. Evolution 61:2469-2479.

d'Errico F, Henshilwood CS, Lawson G, Vanhaeren M, Tillier A-M, Soressi M, Bresson F, Maureille B, Nowell A, Lakarra J, Backwell L, Julien M (2003) Archaeological evidence for the emergence of language, symbolism, and music-an alternative multidisciplinary perspective. J World Prehist 17:1-70.

d'Errico F, Henshilwood CS, Vanhaeren M, van Niekerk K (2005) Nassarius kraussianus shell beads from Blombos Cave: evidence for symbolic behaviour in the Middle Stone Age. J Hum Evol 48:3-24.

Dart RA (1925) Australopithecus africanus: the man-ape of South Africa. Nature 2884:195-199.

de Beaune SA, Coolidge FL, Wynn T (eds) (2009) Cognitive archaeology and human evolution. Cambridge University Press, Cambridge.

Depew DJ (2003) Baldwin and his many effects. In: Weber B, Depew DJ (eds) Evolution and learning: the Baldwin Effect reconsidered. MIT Press, Cambridge, MA.

Dunbar RIM (1998) The social brain hypothesis. Evol Anthropol 6:178-190.

Dunbar RIM (2008) Mind the gap: or why humans aren’t just great apes. Proc Brit Acad 154:403-423.

Dunbar RIM (2010) The social role of touch in humans and primates: behavioural function and neurobiological mechanisms. Neurosci Biobehav Rev 34:260-268.

Ebeling U, Steinmetz H (1995) Anatomy of the parietal lobe: mapping the individual pattern. Acta Neurochir (Wien) 136:8-11.

Eibl-Eibesfeldt I (1989) Human ethology. Aldine de Gruyter, Hawthorne.

Etienne AS, Jeffery KA (2004) Path integration in mammals. Hippocampus 14:180-192.

Feix T, Romero J, Schmiedmayer HB, Dollar AM, Kragic D (2015) The GRASP taxonomy of human grasp types. IEEE Trans Human-Machine Sys 46:66-77.

Feng J, Spence I, Pratt J (2007) Playing an action video game reduces gender differences in spatial cognition. Psychol Sci 18:850-855.

Feblot-Augustin J (1993) Mobility strategies in the late middle Palaeolithic of central Europe and Western Europe: elements of stability and variability. J Anthrop Archaeol 112:211-265.

Feblot-Augustin J (1997) La Circulation des Matières Premières au Paléolithique. Liège: CNRS.

Fletcher PC, Frith CD, Baker SC, Shallice T, Frackowiak RSJ, Dolan RJ (1995) The mind's eye-precuneus activation in memory-related imagery. NeuroImage 2:195-200. 
Foley R (1987) Hominid species and stone-tool assemblages: how are they related? Antiquity 61:380-392.

Foley R, Lahr MM (2003) On stony ground: lithic technology, human evolution, and the emergence of culture. Evol Anthropol 12:109-122.

Foo P, Warren WH, Duchon A, Tarr MJ (2005) Do humans integrate routes into a cognitive map? map- versus landmark-based navigation of novel shortcuts. J Exp Psychol 31:195-215.

Freton M, Lemogne C, Bergouignan L, Delaveau P, Lehéricy S, Fossati P (2014) The eye of the self: precuneus volume and visual perspective during autobiographical memory retrieval. Brain Struct Funct 219:959-968.

Gallagher S (2013) The enactive hand. In: Radman Z (ed) The hand, an organ of the mind: what the manual tells the mental. MIT Press, Cambridge.

Gamble C (1998) Palaeolithic society and the release from proximity: a network approach to intimate relations (Human social evolution). Wild Archaeol 29:426-449.

Gentilucci M, Corballis MC (2006) From manual gesture to speech: a gradual transition. Neurosci Biobehav Rev 30: 949-960.

Golledge RG (2003) Human wayfinding and cognitive maps. In: Rockman M, Steele J (eds) Colonization of unfamiliar landscapes: the archaeology of adaptation. Routledge, London.

Goodale MA, Meenan JP, Bülthoff HH, Nicolle DA, Murphy KJ, Racicot CI (1994) Separate neural pathways for the visual analysis of object shape in perception and prehension. Curr Biol 4:604-610.

Gould SJ, Vrba ES (1982) Exaptation: a missing term in the science of form. Paleobiol 8:4-15.

Grefkes C, Fink GR (2005) The functional organization of the intraparietal sulcus in humans and monkeys. J Anat 207:3-17.

Gunz P, Neubauer S, Maureille B, Hublin J-J (2010) Brain development after birth differs between Neanderthals and modern humans. Curr Biol 20:R921-R922.

Haggard P (2005) Conscious intention and motor cognition. Trends Cogn Sci 9:290-295.

Haggard P, de Boer L (2014) Oral somatosensory awareness. Neurosci Biobehav Rev 47:469-484.

Hagmann P, Cammoun L, Gigandet X, Meuli R, Honey CJ, Wedeen VJ, Sporns O (2008) Mapping the structural core of human cerebral cortex. PLoS Biol 6:1-15.

Hatwell Y (2003) Introduction: touch and cognition. In: Hatwell Y, Streri A, Genatz E (eds) Touching for knowing: cognitive psychology of haptic manual perception. John Benjamins, Amsterdam.

Hatwell Y, Streri, A, Gentaz E (eds) (2003) Touching for knowing: cognitive psychology of haptic manual perception. John Benjamins, Amsterdam.

Hecht EE, Gutman DA, Preuss TM, Sanchez MM, Parr LA, Rilling JK (2013) Process versus product in social learning: comparative diffusion tensor imaging of neural systems for action execution-observation matching in macaques, chimpanzees, and humans. Cereb Cortex 23:1014-1024.

Helbig HB, Ernst MO (2008) Haptic perception in interaction with other senses. In: Grunwald M (ed) Human haptic perception: basics and applications. Birkhäuser Verlag, Basel.

Henshilwood CS, Marean C (2003) The origin of modern human behavior: critique of the models and their test implications. Curr Anthrop 44:627-651.

Hihara S, Notoya T, Tanaka M, Ichinose S, Ojima H, Obayashi S, Fujii N, Iriki A (2006) Extension of corticocortical afferents into the anterior bank of the intraparietal sulcus by tool-use training in adult monkeys. Neuropsychologia 44:2636-2646.

Hills TT, Todd PM, Lazer D, Redish AD, Couzin ID (2015) Exploration versus exploitation in space, mind, and society. Trends Cogn Sci19:46-54. 
Holloway RL (1981) Exploring the dorsal surface of hominoid brain endocasts by stereoplotter and discriminant analysis. Philos Trans R Soc London B Biol Sci 292:155-166.

Hutchins E (1995) Cognition in the wild. MIT Press, Cambridge MA.

Hutto DD, Myin E (2013) Radicalizing enactivism: basic minds without content. MIT Press, Cambridge MA.

Ingber D (2008) Tensegrity-based mechanosensing from macro to micro. Progr Biophys Mol Biol 97:163-179.

Iriki A, Taoka M (2012) Triadic (ecological, neural, cognitive) niche construction: a scenario of human brain evolution extrapolating tool use and language from the control of reaching actions. Philos Trans R Soc London B Biol Sci 367:10-23.

Jirak D, Menz MM, Buccino G, Borghi AM, Binkofski F (2010) Grasping language-a short story on embodiment. Cons Cogn 19:711-720.

Jung RE, Haier RJ (2007) The Parieto-Frontal Integration Theory (P-FIT) of intelligence: converging neuroimaging evidence. Behav Brain Sci 30:135-187.

Kappassov Z, Corrales JA, Perdereau V (2015) Tactile sensing in dexterous robots hands. Rob Auton Sys 74:195-220.

Kelly RL (2013) The Lifeways of Hunter-Gatherers. Cambridge University Press.

Kivell TL, Kibii, JM, Churchill SE, Schmid P, Berger LR (2011) Australopithecus sediba hand demonstrates mosaic evolution of locomotor and manipulative abilities. Science 333:1411-1417.

Lakoff G, Johnson M (2008) Metaphors we live by. University of Chicago Press, Chicago.

Laland KN, Janik VM (2006) The animal cultures debate. Trends Eco Evo 21:542-547.

Land MF (2014) Do we have an internal model of the outside world? Phil Trans R Soc B 369:1-6.

Leroi-Gourhan A (1964) Le geste et la parole. Armand Colin, Paris.

Liu X, Zhan Q (2013) Description of the human hand grasp using graph theory. Med Eng Phys 35:1020-1027.

Lombard M, Haidle MN (2012) Thinking a bow-and-arrow set: cognitive implications of Middle Stone Age bow and stone-tipped arrow technology. Camb Arch J 22:237-264.

Lozano M, Bermúdez de Castro JM, Carbonell E, Arsuaga JL (2008) Non-masticatory uses of anterior teeth of Sima de los Huesos individuals (Sierra de Atapuerca, Spain). J Hum Evol 55:713-728.

Lycett SJ (2011) Most beautiful and most wonderful: those endless stone tool forms. J Evol Psychol 9:143-171.

Machin AJ, Dunbar RIM (2011) The brain opiod theory of social attachment: a review of the evidence. Behav 148:985-1025.

Maguire EA, Burgess N, O’Keefe J (1999) Human spatial navigation: cognitive maps, sexual dimorphism, and neural substrates. Curr Opinion Neurobiol 9:171-177.

Maguire EA, Gadian DG, Johnsrude IS, Good CD, Ashburner J, Frackowiak RSJ, Frith CD (2000) Navigationrelated structural change in the hippocampi of taxi drivers. Proc Natl Acad Sci USA 97:4398-4403.

Maguire EA, Spiers HG, Good CD, Hartley T, Frackowiak RSJ, Burgess N (2003) Navigation expertise and the human hippocampus: a structural brain imaging analysis. Hippocampus 13:250-259.

Maister L, Slater M, Sanchez-Vives MV, Tsakiris M (2015) Changing bodies changes minds: owning another body affects social cognition. Trends Cogn Sci 19:6-12.

Malafouris L (2008) Beads for a plastic mind: the ‘blind man’s stick' (BMS) hypothesis and the active nature of material culture. Camb Arch J 18:401-414.

Malafouris L (2010a) Metaplasticity and the human becoming: principles of neuroarchaeology. J Anthro Sci 88:49-72. 
Malafouris L (2010b) The brain-artefact interface (BAI): a challenge for archaeology and cultural neuroscience. Social Cogn Affective Neurosci 5:264-273.

Malafouris L (2013) How things shape the mind: a theory of material engagement. MIT Press, Cambridge MA.

Maravita A, Iriki A (2004) Tools for the body (schema). Trends Cogn Sci 8:79-86.

Margulies DS, Vincent JL, Kelly C, Lohmann G, Uddin LQ, Biswal BB, Villringer A, Castellanos FX, Milham MP, Petrides M (2009) Precuneus shares intrinsic functional architecture in humans and monkeys. Proc Natl Acad Sci USA 106:20069-20074.

Marino BFM, Gallese V, Buccino G, Riggio L (2012) Language sensorimotor specificity modulates the motor system. Cortex 48:849-856.

Mars R, Jbabdi S, Sallet J, O’Reilly JX, Croxson PL, Olivier E, Noonan MAP, Bergmann C, Mitchell AS, Baxter MG, Behrens TEJ, Johansen-Berg H, Tomassini V, Miller KL, Rushworth MFS (2011) Diffusion-weighted imaging tractography-based parcellation of the human parietal cortex and comparison with human and macaque resting-state functional connectivity. J Neurosci 31:4087-4100.

Marzke MW (1997) Precision grips, hand morphology, and tools. Am J Phys Anthropol 102:91-110.

Marzke MW (2013) Tool making, hand morphology and fossil hominins. Philos Trans R Soc B 368:1-8.

Marzke MW, Marzke RF (2000) Evolution of the human hand: approaches to acquiring, analysing and interpreting the anatomical evidence. J Anat 197:121-140.

Marzke MW, Shackley MS (1986) Hominid hand use in the Pliocene and Pleistocene: evidence from experimental archaeology and comparative morphology. J Hum Evol 15:439-460.

Mattens F (2013) Perception and representation: mind the hand! In: Radman Z (ed) The hand, an organ of the mind: what the manual tells the mental. MIT Press, Cambridge MA.

Menninger K (1992) Number words and number symbols: a cultural history of numbers. Dover, New York.

Meunier D, Lambiotte R, Bullmore ET (2010) Modular and hierarchically modular organization of brain networks. Front Neurosci 4:1-11.

Mountcastle VB (1995) The parietal system and some higher brain functions. Cereb Cortex 5:377-390.

Nadel L, Hardt O (2004) The spatial brain. Neuropsychology 18:473-476.

Napier JR (1956) The prehensile movements of the human hand. J Bone Joint Surg 38B: 902-913.

Neubauer S, Gunz P, Hublin JJ (2009) The pattern of endocranial ontogenetic shape changes in humans. J Anat 215:240-255.

Neubauer S, Gunz P, Hublin J-J (2010) Endocranial shape changes during growth in chimpanzees and humans: A morphometric analysis of unique and shared aspects. J Hum Evol 59:555-566.

Niewoehner WA (2001) Behavioral inferences from the Skhul/Qafzeh early modern human hand remains. Proc Natl Acad Sci USA 98:2979-2984.

Nonaka T (2011) What is the behavior of a C4 quadriplegic mouth calligrapher constant function of? BIO Web of Conferences 1:1-4.

Nonaka T (2013) Motor variability but functional specificity: the case of a C4 tetraplegic mouth calligrapher. Ecol Psychol 25:131-154.

Nonaka T, Bril B (2014) Fractal dynamics in dexterous tool use: the case of hammering behavior of bead craftsmen. J Exp Psychol 40:218-231.

Nonaka T, Bril B, Rein R (2010) How do stone knappers predict and control the outcome of flaking? implications for understanding early stone tool technology. J Hum Evol 59:155-167. 
Nummenmaa L, Tuominen L, Dunbar R, Hirvonen J, Manninen S, Arponen E, Machin A, Hari R, Jääskeläinen IP, Sams M (2016) Social touch modulates endogenous $\mu$-opioid system activity in humans. Neuroimage 138:242-247.

Orban GA, Caruana F (2014) The neural basis of human tool use. Front Psychol 5:1-12.

Orban GA, Claeys K, Nelissen K, Smans R, Sunaert S, Todd JT, Wardak C, Durand JB, Vanduffel W (2006) Mapping the parietal cortex of human and non-human primates. Neuropsychologia 44:2647-2667.

Overmann KA (2015) Teeth, tools and human becoming. J Anthro Sci 93:163-167.

Peer M, Salomon R, Goldberg I, Blanke O, Arzy S (2015) Brain system for mental orientation in space, time, and person. Proc Natl Acad Sci USA 112:11072-11077.

Pelegrin J (2009) Cognition and the emergence of language: a contribution from lithic technology. In: de Beaune SA, Coolidge FL, Wynn T (eds) Cognitive archaeology and human evolution. Cambridge University Press, Cambridge.

Plummer T (2004) Flaked stones and old bones: biological and cultural evolution at the dawn of technology. Y Phys Anthropol 47:118-164.

Ponce de León MS, Bienvenu T, Akazawa T, Zollikofer CPE (2016) Brain development is similar in Neanderthals and modern humans. Curr Biol 26:R665-R666.

Pouydebat E, Gorce P, Coppens Y, Bels V (2009) Biomechanical study of grasping according to the volume of the object: human versus non-human primates. J Biomech 42:266-272.

Prinz J (2009) Is consciousness embodied? In: Robbins P, Aydede M (eds) The Cambridge handbook of situated cognition. Cambridge University Press, Cambridge.

Prinz JJ, Barsalou LW (2000) Steering a course for embodied representation. In: Dietrich E, Markman A (eds) Cognitive dynamics: conceptual change in humans and machines. MIT Press, Cambridge.

Quallo MM, Price CJ, Ueno K, Asamizuya T, Cheng K, Lemon RN, Iriki A (2009) Gray and white matter changes associated with tool-use learning in macaque monkeys. Proc Natl Acad Sci USA 106:18379_ 18384.

Rilling JK (2006) Human and non-human primate brains: are they allometrically scaled versions of the same design? Evol Anthropol 15:65-67.

Rilling JK, Barks SK, Parr LA, Preuss TM, Faber TL, Pagnoni G, Bremner JD, Votaw JR (2007) A comparison of resting-state brain activity in humans and chimpanzees. Proc Natl Acad Sci USA 104:17146-17151.

Rilling JK, Seligman RA (2002) A quantitative morphometrics comparative analysis of the primate temporal lobe. J Hum Evol 42:505-533.

Robbins P, Aydede M (eds) (2009) The Cambridge handbook of situated cognition. Cambridge University Press, Cambridge.

Rochat P (1989) Object manipulation and exploration in 2- to 5-month-old infants. Develop Psychol 25:871-884.

Rochat P (1993) Hand-mouth coordination in the newborn: morphology, determinants, and early development of a basic act. In: Savelsbergh GJP (ed) The development of coordination in infancy. Elsevier, Amsterdam.

Roche RAP, Mangaoang MA, Commins S, O’Mara SM (2005) Hippocampal contributions to neurocognitive mapping in humans: a new model. Hippocampus 15:622-641.

Rolian C, Lieberman DE, Zermeno JP (2011) Hand biomechanics during simulated stone tool use. J Hum Evol $61: 26-41$.

Rushworth MFS, Paus T, Sipila PK (2001) Attention systems and the organization of the human parietal cortex. J Neurosci 21:5262-5271. 
Sakata H, Taira M, Kusunoki M, Murata A, Tanaka Y (1997) The parietal association cortex in depth perception and visual control of hand action. Trends Neurosci 20:350-357.

Saucier DM, Green SM, Leason J, MacFadden A, Bell S, Elias LJ (2002) Are sex differences in navigation caused by sexually dimorphic strategies or by differences in the ability to use the strategies? Behav Neurosci 116:403-410.

Scheperjans F, Eickhoff SB, Hömke L, Mohlberg H, Hermann K, Amunts K, Zilles K (2008) Probabilistic maps, morphometry, and variability of cytoarchitectonic areas in the human superior parietal cortex. Cereb Cortex 18:2141-2157.

Schick K, Toth N, Garufi, G, Savage-Rumbaugh, ES, Rumbaugh D, Sevcik R (1999) Continuing investigations into stone tool-making and tool-using capabilities of a bonobo (Pan paniscus). J Archaeol Sci 26:821-832.

Scott GR, Gibert L (2009) The oldest hand-axes in Europe. Nature 461:82-85.

Scott N, Neubauer S, Hublin JJ, Gunz P (2014) A shared pattern of postnatal endocranial development in extant hominoids. Evol Biol 41:572-594.

Skinner MM, NB Stephens, Tsegai ZJ, Foote AC, Huynh Nguyen N, Gross T, Pahr DH, Hublin JJ, Kivell TL (2015) Human-like hand use in Australopithecus africanus. Science 347: 395-399.

Smith BC (1999) Situatedness/embeddedness. In: Wilson RA, Keil FC (eds) The MIT encyclopedia of the cognitive sciences. MIT Press, Cambridge.

Smith EA (1988) Risk and uncertainty in the "original affluent society”: evolutionary ecology of resource-sharing and land tenure. In: al Ie (ed) Hunters \& Gatherers vol Vol.1.

Sotero RC, Iturria-Medina Y (2011) From Blood Oxygenation Level Dependent (BOLD) signals to brain temperature maps. Bull Math Biol 73:2731-2747.

Spiers HJ, Maguire EA (2006) Thoughts, behaviour, and brain dynamics during navigation in the real world. NeuroImage 31:1826-1840.

Stout D, Chaminade T (2007) The evolutionary neuroscience of tool making. Neuropsych 45:1091-1100.

Stout D, Hecht E, Khreisheh N, Bradley B, Chaminade T (2015) Cognitive demands of lower Paleolithic toolmaking. PloS One 10:1-18.

Suvilehto JT, Glerean E, Dunbar RIM, Hari R, Nummenmaa L (2015) Topography of social touching depends on emotional bonds between humans. Proc Natl Acad Sci USA 112:13811-13816.

Sznajder B, Sabelis MW, Egas M (2012) How adaptive learning affects evolution: reviewing theory on the Baldwin Effect. Evol Biol 39:301-310.

Tallis R (2003) The hand: a philosophical inquiry into human being. Edinburgh University Press, Edinburgh.

Tallis R (2011) Aping mankind: neuromania, Darwinitis and the misrepresentation of humanity. Acumen, Durham.

Tixier J (1967) Procédés d'analyse et questions de terminologie dans l'étude des ensembles industriels du Paléolithique récent et de l'Épipaléolithique en Afrique du Nord-Ouest. In: Bishop WW, Clark JD (eds) Background to evolution in Africa. University of Chicago Press, Chicago.

Tobias PV (1965) Australopithecus, Homo habilis, tool-using and tool-making. S Afr Archaeol Bull 20:167-192.

Tostevin GB (2013) Seeing lithics: a middle-range theory for testing for cultural transmission in the Pleistocene, Oxbow Books, Oakville.

Toth N, Schick KD, Savage-Rumbaugh ES, Sevcik RA, Rumbaugh DM (1993) Pan the tool-maker: investigations into the stone tool-making and tool-using capabilities of a bonobo (Pan paniscus). $\mathrm{J}$ Archaeol Sci 20:81-91.

Turvey MT, Carello C (2011) Obtaining information by dynamic (effortful) touching. Philos Trans R Soc B 366:3123-3132. 
Uomini N, Meyer GF (2013) Shared brain lateralization patterns in language and Acheulean stone tool production: a functional transcranial Doppler ultrasound study. PloS One 8:1-8.

Utevsky AV, Smith DV, Huettel SA (2014) Precuneus is a functional core of the default-mode network. J Neurosci 34:932-940.

Vanduffel W, Fize D, Peuskens H, Denys K, Sunaert S, Todd JT, Orban GA (2002) Extracting 3D from motion: differences in human and monkey intraparietal cortex. Science 298:413-415.

Vankov I, Kokinov B (2013) The role of the motor system in conceptual processing: effects of object affordances beyond response interference. Acta Physiol 143:52-57.

Wadley L (2001) What is cultural modernity? A general view and a South African perspective from Rose Cottage Cave. Camb Archaeol J 11:201-221.

Weissner P (1984) Reconsidering the behavioral basis for style: a case study among the Kalahari San. J Anthropol Archaeol 3:190-234.

Wardak C, Hamed SB, Duhamel JR (2005) Parietal mechanism of selective attention in monkeys and humans. In: Dehaene S, Duhamel JR, Hauser MD, Rizzolatti G (eds) From monkey brain to human brain. MIT Press, Cambridge MA.

Weidenreich F (1941) The brain and its role in the phylogenetic transformation of the human skull. Trans Am Philos Soc XXXI:321-442.

Weissner P (1982) Risk, reciprocity and social influences on !Kung San economics. In: Lee ELR (ed) Politics \& History in Band Societies.

Whallon R (2006) Social networks and information: Non-utilitarian mobility among hunter-gatherers. J Anthrop Archaeol 25:259-270.

Whallon R, Lovis WA, Hitchcock RK (2011) Information and its role in hunter-gatherer bands, vol 5. Ideas, debates and perspectives UCLA/Cotsen Institute of Archaeology Press.

Whitlock JR, Sutherland RJ, Witter MP, Moser M-B, Moser EI (2008) Navigating from hippocampus to parietal cortex. Proc Natl Acad Sci USA 105:14755-14762.

Williams EM, Gordon AD, Richmond BG (2012) Hand pressure distribution during Oldowan stone tool production. J Hum Evol 62:520-532.

Wynn T (1989) The evolution of spatial competence. University of Illinois Press, Chicago.

Wynn T (2000) Symmetry and the evolution of the modular linguistic mind. In: Carruthers P, Chamberlain A (eds) Evolution and the human mind: modularity, language and meta-cognition. Cambridge University Press, Cambridge.

Wynn T (2002) Archaeology and cognitive evolution. Behav Brain Sci 25:389-402.

Wynn T (2014) Commentary on 'extended mind and visuo-spatial integration: three hands for the Neandertal lineage.’ J Anthro Sci 92:291-293.

Wynn T Coolidge FL (2003) The role of working memory in the evolution of managed foraging. Bef Farm 2:1-16.

Zhang S, Li CR (2012) Functional connectivity mapping of the human precuneus by resting state fMRI. NeuroImage 59:3548-3562.

Zilles K, Palomero-Gallagher N (2001) Cyto-, myelo-, and receptor architectonics of the human parietal cortex. NeuroImage 14:S8-S20. 